\title{
The Effect of Aromatherapy on Equine Facial Expression, Heart Rate, Respiratory Tidal Volume and Spontaneous Muscle Contractures in $M$. Temporalis and $M$. Cleidomastoideus
}

\author{
Sofja Kosiara, Adrian Paul Harrison* \\ PAS (Physiology), Faculty of Health and Medical Sciences, University of Copenhagen, Copenhagen, Denmark \\ Email: ^adh@sund.ku.dk
}

How to cite this paper: Kosiara, S. and Harrison, A.P. (2021) The Effect of Aromatherapy on Equine Facial Expression, Heart Rate, Respiratory Tidal Volume and Spontaneous Muscle Contractures in M. Temporalis and M. Cleidomastoideus. Open Journal of Veterinary Medicine, 11, 87-103.

https://doi.org/10.4236/ojvm.2021.112005

Received: December 23, 2020

Accepted: February 22, 2021

Published: February 25, 2021

Copyright $\odot 2021$ by author(s) and Scientific Research Publishing Inc. This work is licensed under the Creative Commons Attribution International License (CC BY 4.0).

http://creativecommons.org/licenses/by/4.0/

\begin{abstract}
The ability to calm horses affects both the safety and well-being of human-horse relationships. However, not many natural calmatives are known, nor the efficacy of these in supporting a state of calmness in horses. This study used both qualitative and quantitative methods to test whether aromatherapy has a measurable and calming effect on equine facial expression and a range of physiological parameters. Nine horses of different ages, genders and breeds were included in a crossover design with humidified essential oils to determine the calming effect of vetiver (Vetiveria zizanioides), spikenard (Nardostachys jatamansi) and roman chamomile (Anthemis nobilis) in relation to water (negative control) and lavender oil (positive control) in horses standing still. The results revealed significantly lowered heart rate $(\mathrm{P}<$ $0.05-<0.001)$ and respiratory tidal volume $(\mathrm{P}<0.05-<0.001)$ for the tested essential oils, while spikenard oil was best at inducing a relaxed facial expression $(60 \%$ of the full-time scale for 8 horses; $75 \%$ of the full-time scale for 7 horses) and roman chamomile oil was statistically most successful at reducing the incidence of spontaneous muscle contractures in $\mathrm{M}$. Temporalis $(\mathrm{P}<$ $0.001)$ and $\mathrm{M}$. Cleidomastoideus $(\mathrm{P}<0.05)$ compared to the other aromatherapy treatments. It is concluded that aromatherapy has both qualitative and quantitative effects on physiological parameters in static horses, and moreover, that essential oils appear to have a calming effect.
\end{abstract}

\section{Keywords}

Horse, Essential Oil, Calmative, Behaviour, Welfare 


\section{Introduction}

Aromatherapy is a branch of herbology, that dates back over 6000 years to ancient Egypt, the Far East and more recently Renaissance Europe [1] [2]. Today, it is one of the fastest growing therapies in the World [1] [2]. Aromatherapy may be beneficial for horses due to the fact that they have an acute sense of smell and that they are prone to environmental stressors [3]. Domesticated horses are exposed to a variety of stressors including new environments, athletic demands, paddock to stable time, and travel [4] [5]. It is reported for humans that aromatherapy leaves individuals feeling uplifted, stimulated, invigorated, or reassured, depending on the oil used [1] [2], however, there is limited research on the use of aromatherapy to calm horses [3] [4] [5] [6].

Lavender oil is the most studied phytotherapeutic for use as an anxiolytic [7] and has documented calming effects in several other species such as rats [8] [9], sheep [10], dogs [11] and humans [12] [13] [14] [15]. Inhalational aromatherapy with lavender essential oil has also been shown to be pain-relieving in human patients in the first days following coronary artery bypass surgery [16]. Popular anxiolytic essential oils are multifarious, and include more than lavender; such as rose, orange, bergamot, lemon, sandalwood, clary sage, roman chamomile and rose-scented geranium [7] [17] but evidence-based documentation is weak.

Horses are capable of relationships with their own species and with other species, just like dogs and humans, using the same signals to communicate [18]. Horses naturally live in family groups that ensure safety and survival [18] [19] and depend on this communication to prevent conflicts and attendant injuries or forced isolation [19]. Communication consists of analysing face and body posture, and avoiding any unpleasantness by using calming signals, displacement behaviour, stress signals and what others have defined as "distance increasing signals" [18]. Such communication is being used to calm not only humans, but animals and other environmental stimuli, as well as forming an essential tool for horses to release their own tension [18].

Therefore, analysis of facial expression and body posture was used in this study to assess how the horses felt about the olfactory stimulation assigned by the aromatherapy without any other imposed external stressor other than them standing still. Measurements of heart rate, respiratory tidal volume and spontaneous muscle contractures in M. Temporalis and M. Cleidomastoideus acted as quantitative support to the behavioural assessment. The null hypothesis tested was that aromatherapy has no effect on facial expression, heart rate, respiratory tidal volume or spontaneous muscle contractures in horses. Furthermore, we did not anticipate any measurable difference between horses when exposed to controls or essential oils treatments, even then applied separately.

\section{Materials and Methods}

\subsection{Animals}

The sample size for this study was based on a previous equine aromatherapy 
study [4]. Each horse was exposed to both control and experimental treatments in a randomized order (see Table 1) using software found on the following website: www.random.org.

The result was that seven castrated male and two intact female horses were recruited from two privately-owned Danish farms, giving a total of nine $(\mathrm{n}=9)$ horses, again based on the sample size predictions from the study by Ferguson and colleagues [4]. The horses lived in stables, where they had an individual stall. Thoughout the experiment, the majority of the horses were out on pasture all day and night $(n=6)$ while the remainder spent their days at pasture and nights in a stall. The horses ranged in age from 13 to 25 years (mean $=17.8$ years) and had very little or no previous experience with aromatherapy. The breeds included Arabian, Connemara, Danish Warmblood, Frederiksborg, Haflinger and Northlands (see Table 2). The inclusion criteria for horses in this study were no visible signs of lameness and no recent Veterinarian issues, furthermore all of the horses were in regular use by their owners.

Copenhagen University, Faculty of Health \& Medical Sciences, PAS not only approved this project but also provided facilities and monitored its progress. The study was carried out in accordance with the Helsinki Declaration. The owners of the horses used, gave their informed consent prior to the start of this study. This study was entirely non-invasive in its nature.

This research was performed on domesticated horses who were in contact

Table 1. The order of the aromatherapy treatments used in this study.

\begin{tabular}{cccccccccc}
\hline Treatment & Amigo Cavalos Copper & Intakt & Max & Noble & Siska & Ville & Zendi \\
\hline Water & 5 & 4 & 4 & 1 & 5 & 4 & 1 & 3 & 2 \\
Lavender & 1 & 2 & 1 & 2 & 4 & 5 & 3 & 2 & 4 \\
Vetiver & 3 & 1 & 3 & 3 & 2 & 3 & 5 & 5 & 3 \\
Spikenard & 2 & 5 & 5 & 4 & 1 & 2 & 4 & 4 & 5 \\
Roman Chamomile & 4 & 3 & 2 & 5 & 3 & 1 & 2 & 1 & 1 \\
\hline
\end{tabular}

Table 2. Profile for each participating horse.

\begin{tabular}{ccccc}
\hline Name & Age $(\mathbf{y r})$ & Sex & Breed & Participating no. \\
\hline Amigo & 25 & Gelding & Connemara & $4\left(12^{\text {th }}\right.$ August 2019) \\
Cavalos & 18 & Gelding & Frederiksborg & $3\left(7^{\text {th }}\right.$ August 2019) \\
Copper & 17 & Mare & Haflinger & $2\left(31^{\text {st }}\right.$ July 2019) \\
Intakt & 16 & Gelding & Arabian & $9\left(21^{\text {st }}\right.$ August 2019) \\
Max & 17 & Gelding & Danish Warmblood & $7\left(19^{\text {th }}\right.$ August 2019) \\
Noble & 13 & Gelding & Danish Warmblood & $6\left(16^{\text {th }}\right.$ August 2019) \\
Siska & 18 & Mare & Connemara & $1\left(13^{\text {th }}\right.$ July 2019) \\
Ville & 18 & Gelding & Northlands & $8\left(20^{\text {th }}\right.$ August 2019) \\
Zendi & 18 & Gelding & Frederiksborg & $5\left(13^{\text {th }}\right.$ August 2019) \\
\hline
\end{tabular}


with people on a daily basis. The feeding behaviour of free-roaming horses suggests that horses intuitively understand how to exploit the physiological and psychological benefits of different plant parts [20] but the behaviour of domesticated horses may differ greatly from horses living in the wild, as a result of their smaller environment, controlled living conditions, interaction with the world of humans, humans themselves, and the demands humans make on domesticated horses.

None of the participating horses had behavioural problems and were reported by their owners as healthy. The experimental design avoided situations that are known to result in extreme fight or flight responses.

\subsection{Experimental Design}

The experimental protocol followed a crossover design with an experimental treatment of humidified essential oils, thus allowing each horse to serve as its own control to account for possible confounding variables such as personality, breed, sex and age. The experiment examined the calming properties of humidified vetiver, spikenard and roman chamomile oil compared to humidified water (negative control) and humidified lavender (positive control), an essential oil that has scientific documented calming properties [7]-[14].

All treatments were performed in a stable with the support of a herd member or alone if the horse was comfortable with that. A horse handler sat in a chair in front of the horse and placed a diffuser (doTERRA Iris, UK) either on the ground between their feet or within half a meter from the ground (in their hand while placed on their leg). Each horse was either tied using two ropes or held by the handler. None of the horses were forced to sniff the vapor but efforts were made to keep the horse nearby during the exposure period.

The aromatherapy treatment was performed for 15 minutes while the horse's behaviour was videotaped (Apple iPhone 11, DK). After each treatment, the heart rate, respiratory tidal volume and spontaneous muscle contractures of the horse were measured for a 10-minute period. These measurements were compared with the average of two 10-minute measurements of the same factors; one measured right after applying the equipment before any other treatment (cf. Baseline \#1), and one measured after all the aromatherapy treatments (cf. Baseline \#2). Between each aromatherapy treatment and before the last baseline measurement, each horse had a 20-minute break in its stall, where it was free to eat hay and walk around.

\subsection{Aromatherapy}

The aromatherapy oils were placed into a portable ultrasonic essential oil diffuser (doTERRA Iris, UK) that creates an ultra-fine, yet substantial mist. The diffuser can contain a maximum of $35 \mathrm{ml} \pm 2 \mathrm{ml}$ of water to which two to three drops of essential oil were added. Excess water and essential oil were discarded after 15 minutes of treatment and the diffuser was cleaned with water. The dif- 
fuser used for the negative control was brand new, had never contained any essential oils and was only used to deliver water throughout the experiment. A second Iris-diffuser was used for the essential oils in this study.

The essential oils used in this study came from doTERRA Europe Ltd. (Altius House, 1 North Fourth St, Milton Keynes, MK9 1DG, UK) who state that their essential oils are gently and carefully distilled from plants that have been patiently harvested at the perfect moment by experienced growers from around the world for ideal extract composition and efficacy. Each batch of essential oils goes through a battery of rigorous and definitive tests to accommodate the Certified Pure Therapeutic Grade ${ }^{\circledR}$ quality protocol.

Lavender essential oil was distilled from Lavandula angustifolia. The oil was tapped in $15 \mathrm{ml}$ amber glass bottles on 13 January 2018 under lot no. 180133 and indicated to expire in January 2023. Vetiver essential oil was distilled from Vetiveria zizanioides and tapped into $15 \mathrm{ml}$ amber glass bottles on 5 February 2018 under lot no. 180367 with indicated expiry in February 2023. Spikenard essential oil was distilled from Nardostachys jatamansi. The oil was tapped in $5 \mathrm{ml}$ amber glass bottles on 28 August 2018 with lot no. 182404 and with indicated expiry in August 2023. Finally, roman chamomile essential oil was distilled from Anthemis nobilis. The oil was tapped into $5 \mathrm{ml}$ amber glass bottles on 1 December 2017 under lot no. 173354 and indicated to expire in December 2022. None of the quality control lot analysis performed by the Aromatic Plant Research Center (230N 1200E Suite 100, Lehi, UT 84043, USA) revealed contaminants or adulteration and all the samples were assessed as meeting the expected chemical profile. Tap water (4600 Køge, DK) was used for control treatments.

\subsection{Equipment}

The heart rate of the horses in this study was measured using a Televet 100 ECG Holter (Jørgen Kruuse A/S, Havretoften 4, 5550 Langeskov, DK) with sensors placed next to the tuberosity of the left and right scapular spine and over the xiphoid process. Televet related software was used to analyse the recorded signals. Indications of spontaneous muscle contractures were measured using Acoustic MyoGraphy with sensors located on M. Temporalis, just above the masseteric nerve, and on M. Cleidomastoideus near the ears. Equipment, sensors and related software were provided by CURO-Diagnostics ApS (Tværledet 33, 2880 Bagsværd, DK). The respiratory tidal volume was measured by an elastic belt placed around the abdomen of the horse, positioned in an outward direction from the third posterior rib. The elastic belt and related software were provided by LEAP Technology ApS (Diplomvej 381, 2800 Kongens Lyngby, DK).

\subsection{Data Processing}

Raw data of heart rate and respiratory tidal volume were examined for measurement bias and 15 random samples were selected from areas without visible bias for each aromatherapy treatment. Results were averaged and are presented 
as the mean \pm standard deviations (SD) or \pm standard errors (SEM).

The data collection of spontaneous muscle contractures was done by counting the number of peaks for each muscle group. Data from essential oils that seemed promising in terms of being calming according to the number of muscle contractions were examined for their spatial and temporal summation with the CURO unit; expressed by the S- and T-score of the combined ESTi Score (CURO-Diagnostics ApS, Bagsværd, DK). A scale of 0 to 10 was used to assess optimal muscle function, where 0 was considered as $0 \%$ optimal and 10 was considered $100 \%$ optimal. This means that a very small amplitude and low frequency is an effective utilization of a muscle because few muscle fibres are being used and they are not being repeatedly re-fired at a high frequency, which is energy demanding. Data were collected as one-minute recordings from five to six minutes inside the 10-minute measurement, i.e. approximately in the middle of the total measurement. S-max was equal to 0.05 while threshold was 0.01 due to the fact that the horses stood still, and muscle contractions occurred from a resting tonus state.

Behavioural assessment was via the analysis of the video recordings done continuously throughout the measurement. The different types of behaviour were either categorized as states where behaviours occurred for an extended duration, or events where behaviours were short in duration and these were counted rather than timed. All behaviour was recorded according to an ethogram (see Table 3). The ethogram comprised facial expression and behavioural patterns which can be divided into categories of calming signals, displacement activities or stress signals according to the definitions by Draaisma [18]. Only signals performed from a position where the horse was standing still were included in this ethogram.

Table 3. Ethogram used for continuous sampling on focal observations during aromatherapy, based on behaviour pattern defined by Draaisma [18].

\begin{tabular}{|c|c|c|c|}
\hline \multicolumn{4}{|r|}{ BODY POSTURE } \\
\hline Low head-neck & Lh & $\mathrm{S}$ & The horse's head and neck are kept at a 45- to 20-degree angle below the back line. \\
\hline Mid-low head-neck & $\mathrm{Ml}$ & $\mathrm{S}$ & The horse's head and neck are kept at a 20- to 5-degree angle below the back line. \\
\hline Horizontal head-neck & $\mathrm{H}$ & $\mathrm{S}$ & The horse's head and neck form a horizontal line in level with the back. \\
\hline Mid-high head-neck & $\mathrm{Mh}$ & $\mathrm{S}$ & The horse's head and neck are kept at a 5- to 20-degree angle above the back line. \\
\hline High head-neck & $\mathrm{Hh}$ & $\mathrm{S}$ & The horse's head and neck are kept at a 20- to 45 -degree angle above the back line. \\
\hline Carried tail & Lct & $\mathrm{E}$ & The tail of the horse is lifted from the hindquarters. \\
\hline Three legged & $\mathrm{Tl}$ & $\mathrm{S}$ & A relaxed horse standing with a hind leg raised and rested on the toe of the hoof. \\
\hline Defecation & $\mathrm{D}$ & $\mathrm{E}$ & The horse defecates. \\
\hline Urination & $\mathrm{U}$ & $\mathrm{E}$ & The horse urinates. \\
\hline \multicolumn{4}{|r|}{ FACIAL EXPRESSION } \\
\hline Almond-shaped eyes & $\mathrm{E}$ & $\mathrm{S}$ & The horse's eyes have an almond shape, which means that the eyes are relaxed and without tension. \\
\hline Rounder eyes & $\operatorname{Re}$ & S & The eyes go from being almond shaped to be rounder as an expression of tension. \\
\hline
\end{tabular}




\section{Continued}

\begin{tabular}{|c|c|c|c|}
\hline Visible white of eyes & We & $\mathrm{S}$ & The eyes are round in shape and the white in the eye is visible as an expression of greater tension. \\
\hline Long nostrils & $\mathrm{N}$ & $\mathrm{S}$ & The horse's nostrils are long in shape, which means the nose is relaxed. \\
\hline Rounder nostrils & $\mathrm{Rn}$ & $\mathrm{S}$ & $\begin{array}{l}\text { The nostrils become rounder with tension. The nostrils may go from long to round and back } \\
\text { again as the horse smells and breathes more heavily, which are not included as rounder nostrils. }\end{array}$ \\
\hline Soft, closed lips & $\mathrm{L}$ & $\mathrm{S}$ & $\begin{array}{l}\text { The horse's mouth is closed with soft lips without wrinkles around the muzzle, which means the } \\
\text { mouth is relaxed. }\end{array}$ \\
\hline Rim of lower lip & $\mathrm{Ll}$ & $\mathrm{S}$ & $\begin{array}{l}\text { The mouth and lower lip are relaxed, allowing the rim of the lower lip to be seen in front of the } \\
\text { upper lip. }\end{array}$ \\
\hline Clenched lips & $\mathrm{Cl}$ & $\mathrm{S}$ & $\begin{array}{l}\text { The mouth is squeezed together making wrinkles around the mouth and lips, which means a } \\
\text { light tension around the mouth. }\end{array}$ \\
\hline Extension of the upper lip & $\mathrm{Ul}$ & $\mathrm{S}$ & There is a light to clearly visible extension of the upper lip as an expression of greater tension. \\
\hline Actively moving ears & $\mathrm{Ae}$ & $\mathrm{E}$ & The ears often move back and forth without pauses with a standstill. \\
\hline \multicolumn{4}{|r|}{ COMMUNICATIVE SIGNALS } \\
\hline Blinking & B & $\mathrm{E}$ & $\begin{array}{l}\text { The horse makes a marked blink where the eyelids are clearly pinched. It does not include fast, } \\
\text { not clearly defined blinking. }\end{array}$ \\
\hline Half closing eyes & $\mathrm{Hc}$ & $\mathrm{E}$ & The eyes are kept half closed for between 2 and 10 seconds. \\
\hline Looking away & $\mathrm{La}$ & $\mathrm{E}$ & $\begin{array}{l}\text { The horse changes his viewing direction. It differs from head turn and neck turn by both ears are } \\
\text { directed in the viewing direction. }\end{array}$ \\
\hline Chewing & $\mathrm{C}$ & $\mathrm{E}$ & $\begin{array}{l}\text { Without food in the mount, the horse is doing the same rotating movement of the lower jaw and } \\
\text { possibly also producing sound. The tongue is not visible. }\end{array}$ \\
\hline Tongue out chewing & $\mathrm{Ct}$ & $\mathrm{E}$ & $\begin{array}{l}\text { The movements made with tongue out chewing are the same as with the chewing described } \\
\text { above. The horse makes a rotating motion with the lower jaw, possibly also producing sound. } \\
\text { However, as he rotates his lower jaw, the tongue is pushed straight forward, out of the mouth, } \\
\text { and then retracted again. This can happen several times in succession. }\end{array}$ \\
\hline Yawning & $\mathrm{Y}$ & $\mathrm{E}$ & $\begin{array}{l}\text { When yawning, a horse opens his mouth for several seconds. The upper lip is retracted, and the } \\
\text { upper and lower teeth are visible. Sometimes the eyes roll back slightly or are half closed. The } \\
\text { ears turn sideways during the yawn. }\end{array}$ \\
\hline Head turn & $\mathrm{Ht}$ & $\mathrm{E}$ & $\begin{array}{l}\text { The horse turns his head to the side while the neck and body remain stationary. The head turns } \\
\text { can be to both sides. One ear points forwards and the other ear points in the direction of the } \\
\text { diffuser/handler. }\end{array}$ \\
\hline Neck turn & $\mathrm{Nt}$ & $\mathrm{E}$ & $\begin{array}{l}\text { The horse turns his head and neck to the side while the torso and feet remain in the same fixed } \\
\text { position. The neck turn can be to both sides. One ear points forwards and the other ear points in } \\
\text { the direction of the diffuser/handler. }\end{array}$ \\
\hline Neck shake & Ns & $\mathrm{E}$ & $\begin{array}{l}\text { The horse's head and neck are between the horizontal and mid-high positions. The horse pushes } \\
\text { his nose forwards. The head and neck of the horse moves in a short, side-to-side shaking motion } \\
\text { a number of times while the horse' torso stays still. The eyes are closed. }\end{array}$ \\
\hline Body shake & Bs & $\mathrm{E}$ & $\begin{array}{l}\text { The horse lowers his head and neck to a horizontal or mid-low position. The nose is extended } \\
\text { forwards slightly. He makes a sideways shaking motion with his body, starting at his head, then } \\
\text { moving to his neck, his torso, and finally flowing into his tail. Sometimes the mouth is slightly } \\
\text { open during this shaking. }\end{array}$ \\
\hline See-saw lowering & Ss & $\mathrm{E}$ & $\begin{array}{l}\text { In } 1 \text { second, the head goes from a high, mid-high, or horizontal position to a mid-low to a low } \\
\text { position. Then, within } 1 \text { to } 5 \text { seconds, the head is lifted back up to a mid-high or high position. }\end{array}$ \\
\hline Sustained lowering & $\mathrm{Sl}$ & $\mathrm{E}$ & $\begin{array}{l}\text { The horse moves his head and neck from a high, mid-high, or horizontal position to a mid-low } \\
\text { to low position in a single second. This pose is held for } 5-25 \text { seconds, after which the head is } \\
\text { lifted to a mid-high to high position again. }\end{array}$ \\
\hline
\end{tabular}


The posture and body language of horses show what they feel and what their intentions are. Emotions and signals come and go according to the feelings of the horse [18]. The more elements observed which reflect tension simultaneously, the higher the tension level of the horse. Thus, how a horse experiences a stimulus and responds to it, can be deduced from its body language (see Table 4). When assessing horses in this way it is important to pay close attention not only to the situation but also the context of the recording because many of the signals also represent a biological function, e.g. grinding and biting as a result of an itch or something similar. It is up to the observer to distinguish the difference between when a horse is performing these behaviours to fulfil a biological function or as part of a commutative sequence [18].

\subsection{Statistical Analysis}

The data were assessed for Gaussian distribution (test for normality), after which $t$-tests were performed (InStat Version 3.0b by GraphPad Software, 2365 Northside Dr. Suite 560, San Diego, CA 92108).

Data were further analysed using the analysis of variance (ANOVA) nonparametric repeated measures, followed by a Wilcoxon matched-pairs signed-ranks comparison for each tested parameter (cf. heart rate, respiratory tidal volume and muscle contractions) to test for statistically significant changes in each treatment compared to that of the average baseline. Differences between means with a $\mathrm{P}<0.05$ were considered significant.

\section{Results}

A power analysis of the collected data revealed that for the essential oils used, the power was between $50 \%$ and $70 \%$, except for vetiver $(<20 \%)$, and that the power of the water versus baseline required a sample size of $>90$ in order for the delta of 1.0 between the two means to become statistically significant.

\subsection{Physiological Measurements}

All four essential oils had similar calming effect ( $-5 \%$ to $-7 \%$ decrease) on the equine heart rate and the same also seemed to be true for equine respiratory tidal

Table 4. State of mind and physical features of the horse. Please note that these colours are reflected in the results presented in Figure 1.

\begin{tabular}{|c|c|c|c|}
\hline State & Head position & Nose, eyes, mouth & Tail \\
\hline Relaxed & All head-neck positions are possible & $\begin{array}{l}\text { Nostrils long, eyes almond-shaped and mouth relaxed. } \\
\text { A rim or corner of the lower lip may be visible }\end{array}$ & Tail is relaxed, hangs loosely \\
\hline Light tension & All head-neck positions are possible & $\begin{array}{l}\text { Nose and/or eyes round, mouth relaxed and loosely } \\
\text { closed }\end{array}$ & Tail is relaxed or carried lightly \\
\hline Medium tension & Mid-high to high head-neck positions & Nose and/or eyes round, mouth clenched & Tail is carried lightly to moderate \\
\hline High tension & High head-neck position & $\begin{array}{l}\text { Nose and eyes round, mouth clenched and possible a } \\
\text { differently shaped upper lip }\end{array}$ & Tail is carried lightly to high \\
\hline
\end{tabular}




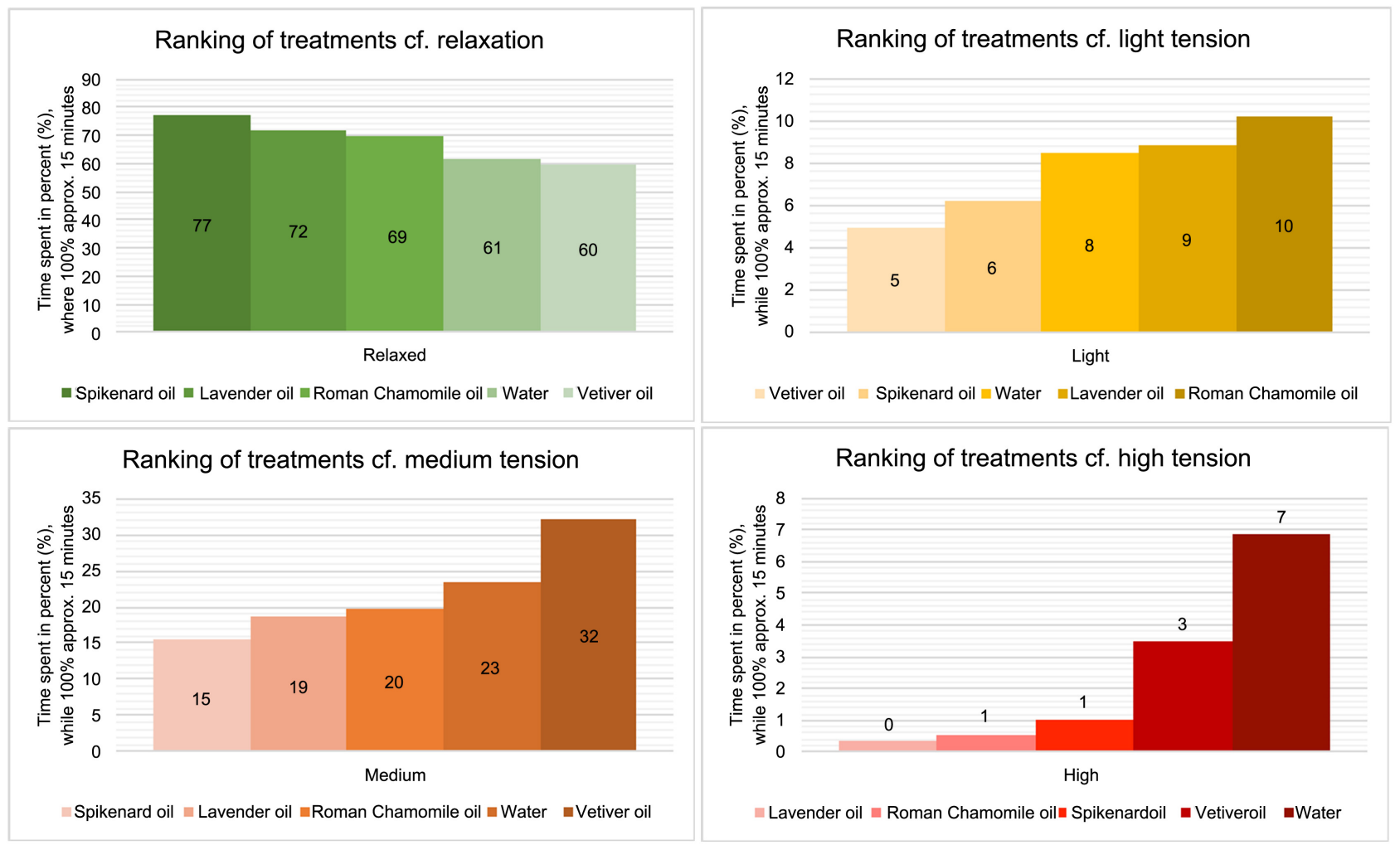

Figure 1. Rank of treatment in relation to average facial expression. From left to right, the treatments were ranked from best to worst in terms of inducing relaxation (green tones) or not inducing light tension (yellow tones), medium tension (orange tones) and high tension (red tones) in percentage.

volume ( $-4 \%$ to $-6 \%$ decrease), at least for three of the essential oil treatments (see Table 5). Roman chamomile resulted in the fewest spontaneous muscle contractions for both muscle groups and with significant differences, while lavender and spikenard only showed a statistic difference for one of each muscle (see Table 5).

Aromatherapy with lavender, spikenard and roman chamomile oil resulted in statistical differences in muscle contractions. Therefore, their S- and T-scores were examined further (see Table 6).

For M. Cleidomastoideus, spikenard was the only essential oil that differed significantly from the baseline with regard to the Acoustic MyoGraphy S- and T-scores, by having a significantly higher frequency $(\mathrm{P}<0.01)$, but roman chamomile had both a significantly lower amplitude $(\mathrm{P}<0.05)$ and lower frequency $(\mathrm{P}<0.01)$ than spikenard along with a significantly lower amplitude $(\mathrm{P}<$ $0.05)$ than lavender, suggesting roman chamomile had a better calming effect on muscle contractions than lavender and spikenard oil. No significant differences were found for the S- and T-scores for M. Temporalis.

\subsection{Behavioural Assessment}

To the authors' knowledge, there are no similar studies and therefore, no similar scales for comparison. Analysis of facial expressions (see Table 4) was used to 
Table 5. Comparison of one-tailed P-values and the percentage change from the mean-baseline across equine heart rate, respiratory tidal volume and muscle contractures for each aromatherapy treatment. ${ }^{*}$ denotes statistical significance of $\mathrm{P}<0.05$; ${ }^{* *}$ denotes statistical significance of $\mathrm{P}<0.01$ and ${ }^{* *}$ denotes statistical significance of $\mathrm{P}<0.001$.

\begin{tabular}{ccccc}
\hline \multirow{2}{*}{$\mathrm{n}=9$} & $\begin{array}{c}\text { Heart rate } \\
(\mathrm{bpm})\end{array}$ & $\begin{array}{c}\text { Tidal volume } \\
(\mathrm{pF})\end{array}$ & \multicolumn{2}{c}{ Muscle contractures (no. of peaks) } \\
\cline { 4 - 5 } & $-5 \%\left(^{* *}\right)$ & $-5 \%\left(^{* * *}\right)$ & $-27 \%$ & $-30 \%\left(^{*}\right)$ \\
\hline Lavender & $-7 \%\left(^{* * *}\right)$ & $-6 \%\left(^{*}\right)$ & $-17 \%$ & $-24 \%$ \\
Vetiver & $-5 \%\left(^{*}\right)$ & $-4 \%\left(^{* *}\right)$ & $-33 \%\left(^{* *}\right)$ & $-20 \%$ \\
Spikenard & & $-39 \%\left(^{* * *}\right)$ & $-34 \%\left(^{*}\right)$ \\
\hline
\end{tabular}

Table 6. $t$-tests performed on the Acoustic MyoGraphy signal as S- and T-scores for baseline (average of measurement before and after all treatments), lavender, spikenard and roman chamomile oil. ${ }^{\star}$ denotes statistical significance of $\mathrm{P}<0.05$; ${ }^{\star \star}$ denotes statistical significance of $\mathrm{P}<0.01$.

\begin{tabular}{ccccc}
\hline & \multicolumn{2}{c}{ S-score } & \multicolumn{2}{c}{ T-score } \\
\cline { 2 - 5 } & M. Temporalis & M. Cleidomastoideus & M. Temporalis & M. Cleidomastoideus \\
\hline Baseline vs Lavender & $6.45>6.19$ & $6.53>6.17$ & $6.81<7.34$ & $7.69>6.71$ \\
Baseline vs Spikenard & $6.45<6.79$ & $6.53>6.21$ & $6.81<7.37$ & $7.69>6.31(* *)$ \\
Baseline vs Roman Chamomile & $6.45>6.05$ & $6.53<6.81$ & $6.81>6.53$ & $7.69>7.46$ \\
Lavender vs Roman Chamomile & $6.19>6.05$ & $6.17<6.81\left(^{*}\right)$ & $7.34>6.53$ & $6.71<7.46$ \\
Lavender vs Spikenard & $6.19<6.79$ & $6.17<6.21$ & $7.34<7.37$ & $6.71>6.31$ \\
Roman Chamomile vs Spikenard & $6.05<6.79$ & $6.81>6.21\left(^{*}\right)$ & $6.53<7.37$ & $7.46>6.31\left(^{* *}\right)$ \\
\hline
\end{tabular}

assess how the horses felt about the aromatherapy. Time spent in seconds was converted to a percentage with $100 \%$ being 896 seconds on average or approximately 15 minutes. Arbitrary values set to $60 \%$ (approx. 9 minutes) and $75 \%$ of the full-time scale (approx. 11 minutes) have been used to compare the percentage of facial relaxation for each aromatherapy treatment (see Table 7) and thereby, assess the effect of the essential oil treatments outlined in this study.

Based on the results in Table 7, vetiver and roman chamomile oil proved to be just a little better than humidified water by inducing one more horse to display a relaxed facial expression for $75 \%$ of the full-time scale. Lavender did better than humidified water for both arbitrary values, but spikenard performed noticeably better than the other treatments.

In order to gain a visually overview, the aromatherapy treatments have been ranked according to their capacity to induce relaxation or avoid tension (see Figure 1). On average, all treatments resulted in a relaxed facial expression for at least $60 \%$ of the full-time scale, while spikenard was the only treatment to result in relaxed body posture for at least $75 \%$ of the full-time scale.

Spikenard resulted in the highest percentage of relaxation with $77 \%$ of the full-time scale and was ranked higher than the controls in relation to avoid light and medium tension. Vetiver oil was the only treatment that was ranked worse 
Table 7. Percentage of horses who had a relaxed facial expression for $60 \%$ and $75 \%$ of the full-time scale.

\begin{tabular}{cccccc}
\hline $\mathrm{n}=9$ & Water & Lavender & Vetiver & Spikenard & Roman Chamomile \\
\hline $60 \%-9 \mathrm{~min}$ & $56 \%(5 / 9)$ & $67 \%(6 / 9)$ & $56 \%(5 / 9)$ & $89 \%(8 / 9)$ & $56 \%(5 / 9)$ \\
$75 \%-11 \mathrm{~min}$ & $33 \%(3 / 9)$ & $56 \%(5 / 9)$ & $44 \%(4 / 9)$ & $78 \%(7 / 9)$ & $44 \%(4 / 9)$ \\
\hline
\end{tabular}

than lavender (positive control) for all four categories and water (negative control) for three of the categories.

\section{Discussion}

To the authors' knowledge, this is the first study to include both qualitative and quantitative data collection methods to investigate the effect of aromatherapy on horses. The measurements selected in this study have assessed the potential calmative effects of humidified essential oils on domesticated horses. This has been the focus of this study since fearful reactions in horses are a major cause of accidents to riders [21], moreover, they can result in uncooperative horses, which at times can even be threatening towards equine practitioners such as veterinarians and farriers [22]. It is also worth mentioning, that fear can have a negative impact on the welfare, reproduction and performance of a horse [23] [24]. This study reveals that aromatherapy has both qualitative and quantitative effects in a positive way on physiological parameters in static horses and moreover, that essential oils have a calming effect.

The facial expression and body language were subsequently assessed according to an ethogram based on published definitions (see Table 3) [18]. Spikenard oil facilitated $17 \%$ longer time with a relaxed facial expression compared to humidified water and proved to be $5 \%$ better than lavender oil in facilitating periods with relaxation (see Figure 1). Lavender oil was only slightly better than spikenard oil to avoid high tension but within such small margins $(-0.7 \%$ difference) that it was reasonable to assume that spikenard was just as good as lavender at avoiding high tension in horses. Roman chamomile oil was on the same level as spikenard and lavender oil at avoiding high tension but was ranked slightly worse than lavender oil in the other three categories. Vetiver oil performed worse than both controls in three out of the four ranking categories (see Figure 1).

The essential oils facilitated a significantly lower heart rate $(-5 \%$ to $-7 \%$ decrease), with lavender $(\mathrm{P}<0.01)$, vetiver $(\mathrm{P}<0.001)$ and roman chamomile oil $(\mathrm{P}<0.001)$ being considered to be very statistically significant. Except for roman chamomile oil, the essential oils resulted in significantly smaller expansion of the ribcage, i.e. the horses breathing was shallower ( $-4 \%$ to $6 \%$ decrease) during aromatherapy, concluded as a sign of relaxation. Lavender $(\mathrm{P}<0.001)$ and spikenard oil $(\mathrm{P}<0.01)$ was considered to be very significant. The essential oils resulted in a reduced incidence of spontaneous muscle contractions, with spikenard $(-33 \%$ decrease, $\mathrm{P}<0.01)$ and roman chamomile oil $(-39 \%$ decrease, $\mathrm{P}<$ 
0.001 ) being considered to be very significant for $\mathrm{M}$. Temporalis, and lavender $(-30 \%$ decrease, $\mathrm{P}<0.05)$ and roman chamomile oil $(-34 \%$ decrease, $\mathrm{P}<0.05)$ being significant for M. Cleidomastoideus. For the S- (i.e. amplitude) and T-scores (i.e. frequency), no significant differences were found for M. Temporalis. However, spikenard oil had a significantly higher frequency than humidified water $(\mathrm{P}<0.01)$ and roman chamomile oil $(\mathrm{P}<0.01)$ which had a significantly lower amplitude than both lavender $(\mathrm{P}<0.05)$ and spikenard oil $(\mathrm{P}<$ 0.05 ) for M. Cleidomastoideus. Thereby, this study supports the literature [25] about roman chamomile oil having a smooth muscle relaxant effect and is better than the other tested oils for this specific purpose.

In terms of limitations, this study, like many others, could not control every possible parameter and as such these might be seen as potential sources of error. In brief, we have used horses that were not trained to work with the diffuser, aromatherapy oils, stand still for longer periods of time etc. Furthermore, we have read the behaviour of the horses based on a published observation [18] and created our own ethogram, the validity of which might be affected by biological functions and personal preferences. It is likewise impossible from the present study to discern the impact of the human handler on the results and indeed the use of the diffuser for all the oil treatments.

Unlike similar scientific studies [4] [5] [6], this study was conducted with the intention of no imposed external stressors. The horses were not trained, neither for receiving aromatherapy nor to stand completely still for an extended period of time with a minimum of action or entertainment in their environment, which could potentially have stressed the horses. Therefore, it was difficult to distinguish whether the tension was due to the scent of essential oil, standing still, having the equipment on, having humans staring at them, being indoors without the herd around them, or simply being bored. All signals were included as communicative responses to aromatherapy but had probably also other triggers.

The behavioural assessment in this study supports observations that signals are used both when a horse is perfectly relaxed or tense [18]. For example, a horse may have a completely relaxed facial expression but still exhibit displacement activities according to the ethogram (see Table 3). In several cases, these signals were listed as communicative but had probably a biological function. Events listed in the ethogram (see Table 3) were found to be far more driven by personal preferences, as documented by the literature [18]. Due to problems comparing results of personal preferences and events being associated with relevant validity bias (i.e. biological function), these results were not included. Analysis of facial expression and body posture is preferable over events for assessing calmness in horses, and trained horses could be interesting to assess in future studies.

Animal testing almost always, involves the presence of humans to a greater or lesser extent. The type of handling and the human involvement in the handling procedures is crucial for the horse' confidence level and responsiveness to treatment [23]. Even when a horse has only been handled previously the experiment, 
one cannot be certain that subsequent decisions made by that horse are not somehow influenced by the body language of the handler [19]. Keeling and colleagues [21] showed that the heart rate significantly increased for both human and horse when a handler was told that an umbrella would be opened as they rode or led a horse past an assistant. Even when an umbrella was never opened, the heart rate of the horse increased when the human "thought" the horse might be frightened [21]. Semin and colleagues [26] discuss the possibility of emotion transfer from human to other species via interspecies chemosignaling of which synanthropic species, such as dogs and horses, seem to be particularly affected due to their long domestication process. Lanata and colleagues [27] found that human body odours induced sympathetic and parasympathetic changes while stimulating horses emotionally. Supporting that emotion transfer happens interspecies via body odours [27] and that physiological synchronicity triggered by emotion chemosignals from humans can unintentionally start a similar physical and emotional reaction in a horse [26], possibly affecting the outcome of a study [19]. Furthermore, it has been proven that horses can discriminate, both behaviourally and physiologically, between positive and negative human facial expressions [27] and between familiar human handlers and human strangers [28]. During the measurements in this study, the horses were handled by two to three humans with different degrees of familiarity, thus emotion chemosignaling, facial expression discrimination and cross-modal discrimination could possibly have affected the current findings. Minimizing or completely removing human influence would be an ideal aim for future studies.

Two identical doTERRA Iris-diffuses were used to perform this study but it can be argued that a separate diffuser for each treatment would have been a more appropriate procedure when the intention was to measure the separate effect of essential oils. Despite rigorous cleaning between trails, there remains a risk of essential oil residues in the diffuser which could have been transferred and possibly influenced the next treatment, although any residue compared with the subsequent treatment oil would have been negligible.

Similarly, there is a risk of carry-over affecting the results when treatments follow each other as they did in this study. One solution could have been to reduce the number of treatments a day. This should, if nothing else, minimize the physiological effect of previous treatments. Since horses have proven several times that they have an excellent memory and recall ability [29], it is difficult to determine whether this solution also minimizes the mental carry-over effect. Based on the current findings, it was assessed that the carry-over effect in this study was minimal if any.

Further improvements to this study could have been made through an alternative choice of data collection methods. The inclusion of such physiological parameters as blood cortisol level or body temperature might be beneficial if they could be obtained without causing stress. In line with humidified essential oils being a non-invasive treatment, the measurement methods selected for this study were likewise non-invasive. One additional technique that is non-invasive 
is thermal imaging, and this could be an obvious extension of the current physiological parameters already outlined in this study. Thermal imaging would for example provide an alternative means of potentially monitoring tense versus relaxed muscles [30]. Finally, an experimental design that allowed the effect of aromatherapy on horses to be tested in horses trained to be led into a reassuring but confined space, where the air was filled with the scent of a given essential oil, would further reduce any potential interference from humans or objects such as diffusers.

\section{Conclusion}

Based on both qualitative and quantitative methods, it is concluded that aromatherapy facilitates a greater degree of facial relaxation, a significantly lower heart rate and respiratory tidal volume along with significantly fewer spontaneous muscle contractures in domesticated horses. The biggest difference between the selected essential oils appeared to be in terms of facial expression and spontaneous muscle contractions. Spikenard oil was best at achieving the longest recorded time with a relaxed facial expression and that was true for the majority of the participating horses, i.e. $78 \%$ of the horses tested revealed that spikenard oil inspired a relaxed body posture for at least $75 \%$ of the full-time scale, while roman chamomile oil was better than the other essential oils in terms of achieving muscle relaxation. Thus, spikenard and roman chamomile oil appear to be better at calming horses than lavender oil, which has otherwise been the best-documented essential oil to date. A larger and more detailed study of these promising preliminary findings is now advocated.

\section{Author Contributions}

Project design was arrived at by SK and APH. Data analysis was performed by SK and AH. All authors contributed to the interpretation and writing of this manuscript.

\section{Acknowledgements}

The authors would like to thank CURO-Diagnostics ApS and LEAP Technology ApS for providing equipment, and all the horse owners for their generous help.

\section{Declaration}

$\mathrm{APH}$ is in the process of establishing a company to produce and market the Acoustic MyoGraphy (CURO-Diagnostics ApS).

\section{Conflicts of Interest}

The authors declare no conflicts of interest regarding the publication of this paper.

\section{References}

[1] Thomas, D.V. (2002) Aromatherapy: Mythical, Magical or Medicinal? Holistic 
Nursing Practice, 17, 8-16. https://doi.org/10.1097/00004650-200210000-00005

[2] Moss, M., Howarth, R., Wilkinson, L. and Wesnes, K. (2006) Expectancy and the Aroma of Roman Chamomile Influence Mood and Cognition in Healthy Volunteers. The International Journal of Clinical Aromatherapy, 16, 63-73. https://doi.org/10.1016/j.ijat.2006.04.002

[3] Baldwin, A.L. and Chea, I. (2018) Effect of Aromatherapy on Equine Heart Rate Variability. Journal of Equine Veterinary Science, 68, 46-50. https://doi.org/10.1016/j.jevs.2018.05.213

[4] Ferguson, C.E., Kleinman, H.F. and Browning, J. (2013) Effect of Lavender Aromatherapy on Acute-stressed Horses. Journal of Equine Veterinary Science, 33, 67-69. https://doi.org/10.1016/j.jevs.2012.04.014

[5] Heitman, K., Rabquer, B., Heitman, E., Streu, C. and Anderson, P. (2018) The Use of Lavender Aromatherapy to Relieve Stress in Trailered Horses. Journal of Equine Veterinary Science, 63, 8-12. https://doi.org/10.1016/j.jevs.2017.12.008

[6] Pouteraud, A., Guilloteau, L., Gros, C., Lobstein, A., Meziani, S., Steyer, D., Moisan, M., Foury, A. and Lansade, L. (2018) Lavender Essential Oil Decreases Stress Response of Horses. Environmental Chemistry Letters, 16, 539-544.

https://doi.org/10.1007/s10311-017-0681-8

[7] Lv, X.N., Liu, Z.J., Zhang, H.J. and Tzeng, C.M. (2013) Aromatherapy and the Central Nerve System (CNS): Therapeutic Mechanism and its Associated Genes. Current Drug Targets, 14, 872-879. https://doi.org/10.2174/1389450111314080007

[8] Tsang, H.W.H., Lo, S.C.L., Chan, C.C.H., Ho, T.Y.C., Fung, K.M.T., Chan, A.H.L. and Au, D.W.H. (2012) Neurophysiological and Behavioural Effects of Lavender Oil in Rats with Experimentally Induced Anxiety. Flavour and Fragrance Journal, 28, 168-173. https://doi.org/10.1002/ffj.3148

[9] Coelho, L.S., Correa-Netto, N.F., Masukawa, M.Y., Lima, A.C., Maluf, S., Linardi, A. and Santos-Junior, J.G. (2018) Inhaled Lavandula angustifolia Essential Oil Inhibits Consolidation of Contextual-Nut Not Tone-Fear Conditioning in Rats. Journal of Ethnopharmacology, 215, 34-41. https://doi.org/10.1016/j.jep.2017.12.038

[10] Hawken, P.A.R., Fiol, C. and Blache, D. (2012) Genetic Differences in Temperament Determine whether Lavender Oil Alleviates or Exacerbates Anxiety in Sheep. Physiology \& Behavior, 105, 1117-1123. https://doi.org/10.1016/j.physbeh.2011.12.005

[11] Graham, L., Wells, D.L. and Hepper, P.G. (2004) The Influence of Olfactory Stimulation on the Behavior of Dogs Housed in a Rescue Shelter. Applied Animal Behaviour Science, 91, 143-153. https://doi.org/10.1016/j.applanim.2004.08.024

[12] Cruz, A.B., Kim, T.H. and Park, S.B. (2011) Effects of Lavender (Lavandula angustifolia Mill.) and Peppermint (Mentha cordifolia Opiz.) Odors on Anxiety and Sport Skill Performance. Asia Life Sciences, 20, 323-329.

[13] Liu, C.-W., Wang, C.-S., Chuang, K.-J., Lo, C.-C. and Chen, C.-T. (2013) Electroencephalographic Study of Essential Oils for Stress Relief. Applied Mechanics and Materials, 43, 1085-1088.

https://doi.org/10.4028/www.scientific.net/AMM.437.1085

[14] Kusumawardani, S.R., Fitri, L.L. and Suprijanto (2017) Theta Brainwave Activity as the Response to Lavender (Lavendula angustifolia) Aromatherapy Inhalation of Postgraduate Students with Acasdemic Stress Condition. IOP Conference Series. Materials Science and Engineering, 180, Article ID: 012271. https://doi.org/10.1088/1757-899X/180/1/012271

[15] Tabatabaeichehr, M. and Mortazavi, H. (2020) The Effectiveness of Aromatherapy in the Management of Labor Pain and Anxiety: A Systematic Review. Ethiopian 
Journal of Health Sciences, 30, 449-458. https://doi.org/10.4314/ejhs.v30i3.16

[16] Bikmoradi, A., Seifi, Z., Bazrafshan, M., Poorolajal, J., Araghchian, M., Kashfi, S.H., Kavi, E. and Jokar, M. (2018) Effect of Inhalation Aromatherapy with Lavender Essential Oil on Pain Severity of Patients after Coronary Artery Bypass surgery: A Single-Blind Randomized Clinical Trial. Journal of Clinical and Diagnostic Research, 12, LC01-LC05.

[17] Xu, J., et al. (2015) Vanillin-Induced Amelioration of Depression-Like Behaviors in Rats by Modulating Monoamine Neurotransmitters in the Brain. Psychiatry Research, 225, 509-514. https://doi.org/10.1016/j.psychres.2014.11.056

[18] Draaisma, R. (2018) Language Signs and Calming Signals of Horses-Recognition and Application. Strous, S., Translator, Taylor \& Francis Group, Abingdon-on-Thames. https://doi.org/10.1201/9781315265667

[19] Ladewig, J. (2019) Body Language: Its Importance for Communication with Horses. Journal of Veterinary Behavior, 29, 108-110. https://doi.org/10.1016/j.jveb.2018.06.042

[20] Crowell-Davis, S.L., Houpt, K.A. and Carnevale, J. (1984) Feeding and Drinking Behavior of Mares and Foals with Free Access to Pasture and Water. Journal of Animal Science, 60, 883-889. https://doi.org/10.2527/jas1985.604883x

[21] Keeling, L.J., Jonare, L. and Lanneborn, L. (2009) Investigating Horse-Human Interactions: The Effect of a Nervous Human. Veterinary Journal, 181, 70-71. https://doi.org/10.1016/j.tvjl.2009.03.013

[22] Houpt, K.A. and Mills, D.S. (2006) Why Horse Behaviour Is Important to the Equine Clinician. Equine Veterinary Journal, 38, 386-387. https://doi.org/10.2746/042516406778400538

[23] Forkman, B., Boissy, A., Meunier-Salaün, M.-C., Canali, E. and Jones, R.B. (2007) A Critical Review of Fear Tests Used on Cattle, Pigs, Sheep, Poultry and Horses. Physiology \& Behavior, 92, 340-374. https://doi.org/10.1016/j.physbeh.2007.03.016

[24] Christensen, J.W., Malmkvist, J., Nielsen, B.L. and Keeling, L.J. (2008) Effects of a Calm Companion on Fear Reactions in Naive Test Horses. Equine Veterinary Journal, 40, 46-50. https://doi.org/10.2746/042516408X245171

[25] Sándor, Z., Mottaghipisheh, J., Veres, K., Hohmann, J., Bencsik, T., Horváth, A., Kelemen, D., Papp, R., Barthó, L. and Csupor, D. (2018) Evidence Supports Tradition: The in Vitro Effects of Roman Chamomile on Smooth Muscles. Frontiers in Pharmacology, 9, 323. https://doi.org/10.3389/fphar.2018.00323

[26] Semin, G.R., Scandurra, A., Baragli, P., Lanatà, A. and D’Aniello, B. (2019) Interand Intra-Species Communication of Emotion: Chemosignals as the Neglected Medium. Animal, 9, 887. https://doi.org/10.3390/ani9110887

[27] Smith, A.V., Proops, L., Grounds, K., Wathan, J. and McComb, K. (2015) Functionally Relevant Responses to Human Facial Expressions of Emotion in the Domestic Horse (Equus caballus). Biology Letters, 12, Article ID: 20150907. https://doi.org/10.1098/rsbl.2015.0907

[28] Proops, L. and McComb, K. (2012) Cross-Modal Individual Recognition in Domestic Horses (Equus caballus) Extends to Familiar Humans. Proceedings of the Royal Society B: Biological Sciences, 279, 3131-3138.

https://doi.org/10.1098/rspb.2012.0626

[29] Murphy, J. (2009) Assessing Equine Prospective Memory in a Y-Maze Apparatus. Veterinary Journal, 181, 24-28. https://doi.org/10.1016/j.tvjl.2009.03.028

[30] Tunley, B.V. and Henson, F.M.D. (2004) Reliability and Repeatability of Thermo- 
graphic Examination and the Normal Thermographic Image of the Thoracolumbar Region in the Horse. Equine Veterinary Journal, 36, 306-312.

https://doi.org/10.2746/0425164044890652 